\title{
Fusion-fission probabilities, cross sections, and structure notes of superheavy nuclei
}

\author{
Michat Kowal ${ }^{1, \text { a }}$, Tomasz Cap ${ }^{1}$, Piotr Jachimowicz ${ }^{2}$, Janusz Skalski ${ }^{1}$, \\ Krystyna Siwek-Wilczyńska ${ }^{3}$, and Janusz Wilczyński ${ }^{1, \mathrm{~b}}$ \\ ${ }^{1}$ National Centre for Nuclear Research, Hoża 69, 00-681 Warsaw, Poland \\ ${ }^{2}$ Institute of Physics, University of Zielona Góra, Szafrana 4a, 65516 Zielona Góra, Poland \\ ${ }^{3}$ Institute of Experimental Physics, Faculty of Physics, University of Warsaw, Warsaw, Poland
}

\begin{abstract}
Fusion - fission probabilities in the synthesis of heaviest elements are discussed in the context of the latest experimental reports. Cross sections for superheavy nuclei are evaluated using the "Fusion by Diffusion" (FBD) model. Predictive power of this approach is shown for experimentally known $\mathrm{Lv}$ and $\mathrm{Og}$ isotopes and predictions given for $Z=119,120$. Ground state and saddle point properties as masses, shell corrections, pairing energies, and deformations necessary for cross-section estimations are calculated systematically within the multidimensional microscopicmacroscopic method based on the deformed Woods-Saxon single-particle potential. In the frame of the FBD approach predictions for production of elements heavier than $Z=118$ are not too optimistic. For this reason, and because of high instability of superheavy nuclei, we comment on some structure effects, connected with the $K$-isomerism phenomenon which could lead to a significant increase in the stability of these systems.
\end{abstract}

\section{Introduction}

The uncharted region of the $Z-N$ plane can answer many questions of fundamental importance for science. One of them is: "What can be the largest possible atomic number $Z$ of an atomic nucleus?". The current answer was gained thanks to the tremendous advance in experimental studies achieved in recent years [1-5] via fusion-evaporation reactions, once the element $Z=118$ has been synthesized [6,7]. The question whether we can go still further waits for an answer as attempts to go beyond $Z=118$ hit two obstacles: (i) a difficulty or impossibility of making targets from Es and heavier actinides, and (ii) reactions with heavier projectiles like ${ }^{50} \mathrm{Ti},{ }^{54} \mathrm{Cr},{ }^{58} \mathrm{Fe}$, and ${ }^{64} \mathrm{Ni}$, did not produce any ERs up to now. The cross sections for the synthesis of heaviest elements are of the order of picobarns or even femtobarns. It happens because of a very strong hindrance occurring in the fusion process itself. The fusion is a highly dissipative process in which the fusing system, staring from a touching configuration must passage over the saddle point into the configuration of the compound nucleus.

The main issue therefore boils down to the problem of motion in the potential surface with a local minimum (representing the meta-stable state) and the barrier protecting this minimum which one should overcome to fuse two nuclei. Taking into account the influence

\footnotetext{
a e-mail: michal.kowal@ncbj.gov.pl

${ }^{\mathrm{b}}$ Deceased
}

(C) The Authors, published by EDP Sciences. This is an Open Access article distributed under the terms of the Creative Commons Attribution License 4.0 (http://creativecommons.org/licenses/by/4.0/). 
of the environment requires addition of the damping force and the stochastic fluctuations. In the case of so called "fusion-by-diffusion" model (FBD) [8], the stochastic process of shape fluctuation leads to the overcoming of the saddle point. The fluctuating force, which is de facto caused by shape fluctuations, is assumed as a white noise to obtain the solution of the Smoluchowski diffusion equation in the deformation space along the fission valley. Thus, basically we touch the famous Kramers problem [9]; the relaxation from the meta-stable state with the assumption of the white noise and with damping proportional to the velocity. Induced or spontaneous relaxation from the meta-stable state via fluctuating potential barriers occurs in many branches of physics: phase transitions, bi-stability in quantum optic, spontaneous emission from excited states, electron transport in semiconductors, kinetics of chemical reactions, etc. [10].

The fundamental assumption which allows to investigate the mechanism of the formation of superheavy compound nuclei is the Bohr hypothesis, which states that the synthesis of a compound system can be treated as a Markow-type process (a stochastic process without any memory effect). This implies that the exit channel is completely independent of the entrance channel and both are independent of the intermediate stage of the reaction leading to the compound nucleus. The Bohr hypothesis can be justified mainly due to the different time scales of the particular stages. According to this hypothesis the total probability for the synthesis of a new superheavy element can be factorized into three independent ingredients:

$$
P_{\text {tot }}=P_{\text {cap }} \times P_{\text {fus }} \times P_{\text {sur }},
$$

where $P_{\text {cap }}$ stands for the probability of overcoming the Coulomb barrier, called the "capture process", $P_{f u s}$ is the formation probability that the nucleus, starting from the touching configuration, will finish up with a compound nuclear shape, and $P_{s u r}$ is the probability that the compound nucleus will survive against fission. The cross sections for the synthesis of superheavy nuclei are dramatically small because the fusion probability $P_{f u s}$ is hindered (in some reactions even by several orders of magnitude) due to the fact that the saddle configuration of the heaviest compound nuclei is much more compact than the configuration of two colliding nuclei at sticking. Survival probabilities, $P_{\text {sur }}$, are in turn extremely sensitive to the height of the fission barrier, especially in case of hot fusion reactions, because at each step of the deexcitation cascade the competition between neutron emission and fission strongly depends on the difference of energy thresholds for these two decay modes.

Low probabilities of for superheavy nuclei production and their high instability makes it worthwhile to search for long-lived exotic configurations. Obvious candidates are high- $K$ isomers or ground states with a high $K$ quantum number.

\section{Model fusion-by-diffusion with macroscopic-microscopic input}

The FBD model [8] in its extended version [11] serves to calculate cross sections for the synthesis of superheavy nuclei. The extension consists in including the angular momentum dependence of all probabilities of successive stages in Eq. (1). In other words, for each angular momentum $(\ell)$ the partial evaporation residue cross section $\sigma_{E R}(\ell)$ for production of a given final nucleus in its ground state is factorized as the product of the partial capture cross sections:

$$
\sigma_{E R}(\ell)=\hbar^{2} \pi \sum_{l=0}^{\infty}(2 l+1) P_{c a p}(\ell) P_{f u s}(\ell) P_{\text {sur }}(\ell),
$$


where $\hat{\pi}^{2}=\hbar^{2} / 2 \mu E_{c . m}$. is the wavelength ( $\mu$ is the reduced mass of the colliding system and $E_{c . m}$. is the energy in the center of mass).

It is assumed in the FBD model that after the contact of the two nuclei, a neck between them grows rapidly at an approximately fixed mass asymmetry and constant length of the system. This "neck zip" is expected to carry the system towards the bottom of the asymmetric fission valley. This is the "injection point" from where the system starts its climb uphill over the saddle in the process of thermal fluctuations in the shape degrees of freedom. Theoretical justification of the above picture of fast zipping the neck was given in Ref. [12], where the later stage of the stochastic climb uphill was described by solving the two-dimensional Langevin equation. Theoretical location of an effective injection point can be deduced from this model [12]. Also in a modified fusion-by-diffusion model [13] the location of the injection point was estimated theoretically.

Competition between neutron emission and fission can be resolved in the spirit of the transition state theory with the help of the well known formulas. All details regarding the calculations of the survival probability, $P_{\text {sur }}$, can be found in Ref. [11]. In case of calculating multiple evaporation ( $\mathrm{x} n$ ) channels a simplified algorithm [14], avoiding the necessity of using the Monte Carlo method, was applied. The most sensitive point here is the knowledge of the fission barrier height. The most important in fission-barrier calculations is its reliability which, to large extent, is based on the model used for calculating energy of a nucleus as a function of deformation. Energy maps are necessary to appreciate fission barriers. In our case multi-dimensional energy landscapes are calculated within the Microscopic-Macroscopic (MM) model based on the deformed Woods-Saxon potential [15]. The Strutinski shell and pairing correction [16] is taken for the microscopic part. For the macroscopic part we use the Yukawa plus exponential model [17] with parameters specified in [18]. Thus, all parameter values are kept exactly the same as in all recent applications of the model to heavy and superheavy nuclei. Mononuclear shapes can be parameterized via spherical harmonics, $\mathrm{Y}_{l m}(\vartheta, \varphi)$, by the following equation of the nuclear surface:

$$
R(\vartheta, \varphi)=c(\{\beta\}) R_{0}\left(1+\sum_{\lambda=1}^{\infty} \sum_{\mu=-\lambda}^{+\lambda} \beta_{\lambda \mu} \mathrm{Y}_{\lambda \mu}\right),
$$

where $c(\{\beta\})$ is the volume-fixing factor and $R_{0}$ is the radius of a spherical nucleus. This parameterization has its limitations; certainly, it is not suitable for too elongated shapes. However, for moderately deformed saddle points in superheavy nuclei it excellently reproduces all shapes generated by other parametrizations, e.g. by Ref. [19], as we checked in numerous tests. For nuclear ground states it is possible to confine the analysis to axiallysymmetric shapes, with the expansion truncated at $\beta_{80}$.

All details of calculations of ground-state and saddle-point properties as masses, shell corrections, pairing energies, and deformations can be found in Ref. [20, 21]. However, in cited papers all tables are limited to even-even nuclei. This is why the fission barrier heights for other nuclei have been calculated separately by adding the quasiparticle energy, $E_{q}=\sqrt{\left(\varepsilon_{q}-\lambda\right)^{2}+\Delta^{2}}$, where $\varepsilon_{q}$ is the energy of the odd nucleon state $q, \lambda$ is the Fermi energy and $\Delta$ is the pairing-gap energy. Calculations have been performed without blocking.

It should be emphasized that in the frame of this method the largest ground-state shell effect $(\sim 9 \mathrm{MeV})$ is observed for the semi-magic nucleus ${ }^{270} \mathrm{Hs}(Z=108, N=162)$. The second minimum $(\sim 7 \mathrm{MeV})$ of the shell correction is located around the nucleus $Z=114$ and $N=184$. When superposed with the weakly deformation-dependent macroscopic part, this component is largely responsible for the emergence of global minima in superheavy nuclei. Concerning the fission barriers, one obtains three areas with clearly raised barriers: around $N \approx 152, N=162$, and $N \approx 180$. There is also a region of low barriers around $N=170$. 

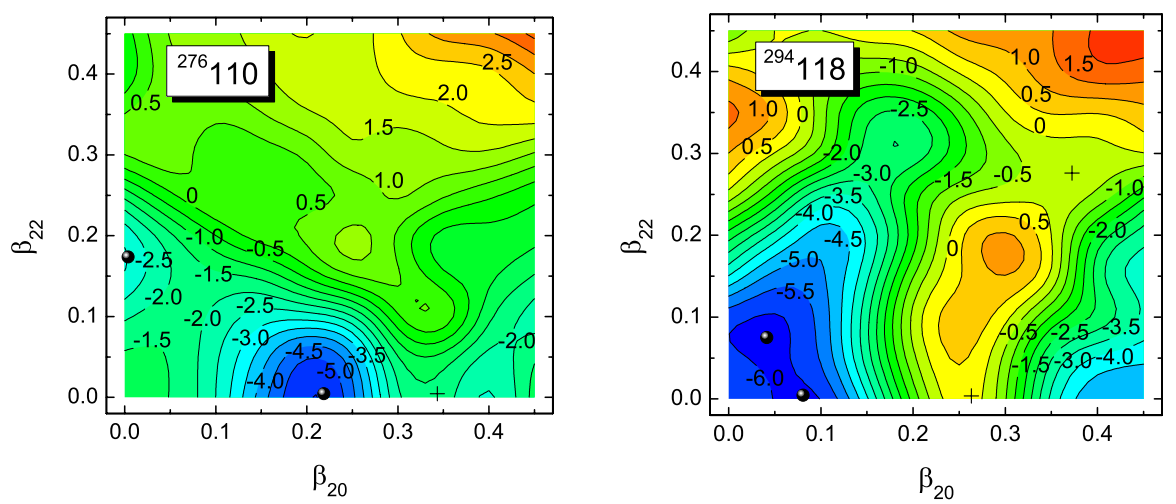

Figure 1. Energy surfaces for two nuclei known experimentally. See text for details.

Finally, let us emphasize that our calculated first and second fission barriers in actinides are in a very good agreement with the experimental/empirical data: the root mean square deviation is $0.7 \mathrm{MeV}$ [22] for the second, and $0.5 \mathrm{MeV}$ [20] for the first fission barriers. In our opinion, such agreement justifies using our model for predictions in heavier nuclei.

\section{Predictions for the synthesis cross sections of $Z=114-120$}

Since the inclusion of triaxiality is absolutely necessary in the superheavy region, we start with showing in Fig. 1 two landscapes in the $\left(\beta_{20}, \beta_{22}\right)$ plane of experimentally known nuclei: ${ }^{276} 110$ and ${ }^{294} 118$. The potential energy surface calculated in the multi-dimensional space, is projected in Fig. 1 on the $\left(\beta_{20}, \beta_{22}\right)$ plane. This means that it is shown as a function of $\beta_{20}$, $\beta_{22}$, but at each given $\left(\beta_{20}, \beta_{22}\right)$ point it is minimized in the remaining degrees of freedom. Here the minimization has been performed over $\beta_{30}, \beta_{40}, \beta_{42}, \beta_{44}, \beta_{50}, \beta_{60}, \beta_{70}$, and $\beta_{80}$. The energy on the map is normalized in such a way that its macroscopic part is put equal to zero at the spherical shape of a nucleus. In ${ }^{276} 110$ one can see two minima: prolate - a prolate ground state and a prolate superdeformed one, see left panel of Fig. 1. The map shows also the axial fission saddle. The nucleus $Z=118, N=176$ is nearly spherical as can be seen in right panel of Fig. 1. There is also a secondary oblate superdeformed minimum. Competition between triaxial and axial saddles is clearly visible.

In the FBD model there is one fit parameter, namely $s_{i n j}$, which is defined as the excess of the total length of the combined system over the length of the initial system (at the touching configuration) when the neck-zip process brings the system to the asymmetric fission valley. Based on the nuclear masses and fission barriers deduced from the energy maps (as shown above for ${ }^{276} 110$ and ${ }^{294} 118$ ) one can adjust this parameter to the experimental synthesis cross section at the maximum of a given (xn) excitation function. Details of this fit procedure with the specification of reaction data used can found in Ref. [23]. The compilation of the so-deduced $s_{i n j}$ values is displayed in Fig. 2 as a function of the kinetic energy excess, $E_{c . m}-B_{0}$, above the Coulomb barrier, $B_{0}$, in the case of two sets of theoretical input data: based on the deformed Woods-Saxon model [20,21] described here (left panel) and assuming the fission-barrier heights [24] and the ground-state masses [25] of P. Möller et al. (right panel).

Based on what we known from the dynamics of nucleus-nucleus collisions one can expect that the injection distance will increase with the decreasing energy: $E_{c . m}$. $-B_{0}$. It is exactly the case if we are using our data. Such a very good correlation between the $s_{i n j}$ values and 

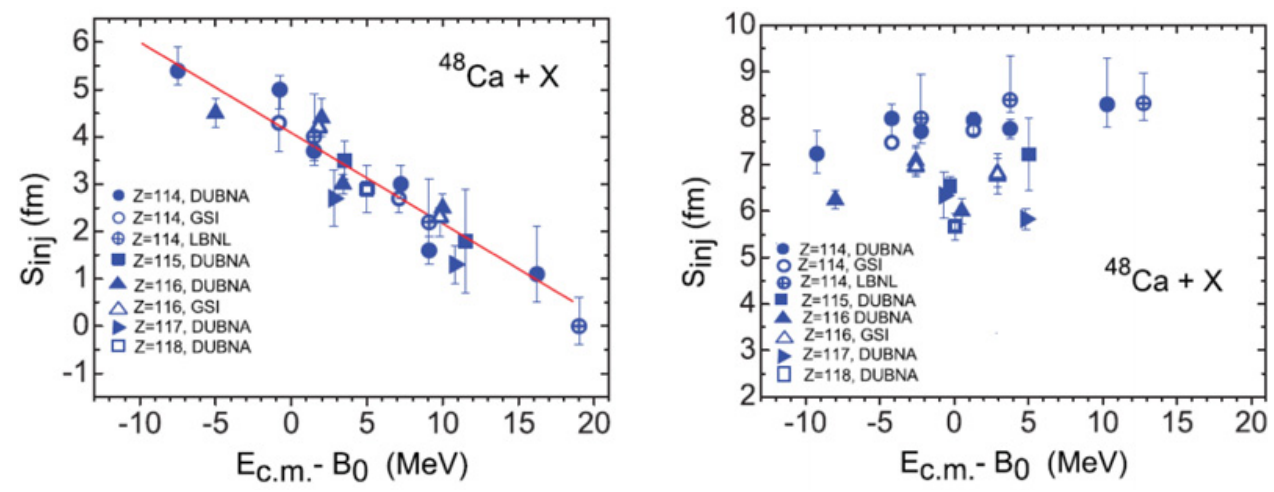

Figure 2. Systematics of the injection-point distance, $s_{i n j}$, as a function of the kinetic energy excess determined using our MM approach [20,21] on the left, and with P. Möller's compilation of nuclear data $[24,25]$ on the right.
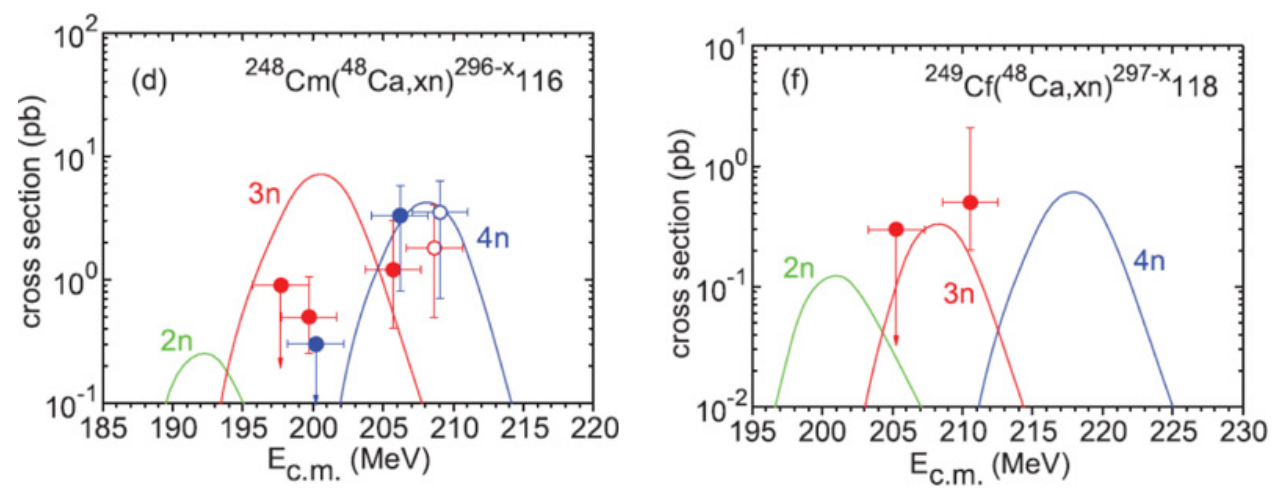

Figure 3. Energy dependence of the cross section for synthesis of superheavy nuclei in hot fusion reactions. Full circles represent data for $3 n, 4 n$, and $5 n$ reaction channels obtained in Dubna experiments for elements $Z=116$ and $Z=118$. Open circles represent data taken at GSI.

the corresponding energies $E_{c . m} .-B_{0}$ can be viewed as an argument in favour of our MM results because such a striking correlation would be very unlikely if the theoretical barrier heights were inconsistent with experimental values. In contrast, the right panel of this figure demonstrates the evident inconsistency of the set of $s_{i n j}$ values obtained for the barriers of Ref. [24] in which the individual points seem to be almost randomly scattered and do not show any correlation with energy.

In Ref. [23] we calculated the excitation functions for known superheavy elements starting from Flerovium up to Oganesson, using results of our MM model. In Fig. 3 we show two examples, namely ${ }^{292} \mathrm{Lv},{ }^{293} \mathrm{Lv},{ }^{294} \mathrm{Lv}$ and ${ }^{293} \mathrm{Og},{ }^{294} \mathrm{Og},{ }^{295} \mathrm{Og}$. Taking into account that the fission barriers could not be calculated more precisely than up to $0.5 \mathrm{MeV}$, the obtained agreement with experimental data can be considered satisfactory.

Then we want to give predictions for currently unknown superheavy elements $Z=119$ and $Z=120$. Particulary interesting is the nucleus ${ }^{302} 120$, as two unsuccessful attempts to produce it have already taken place at GSI, providing a one-event cross-section limit of $0.58\left(\begin{array}{l}134 \\ 48\end{array}\right) \mathrm{pb}$ [26] or $90 \mathrm{fb}$ in [27], and in Dubna [28], providing the limit of $400 \mathrm{fb}$. The crosssection estimates [29] do not support a possibility of an easy production of this superheavy isotope in the laboratory. It is worth noting that with the barrier of the order of $10 \mathrm{MeV}$, 

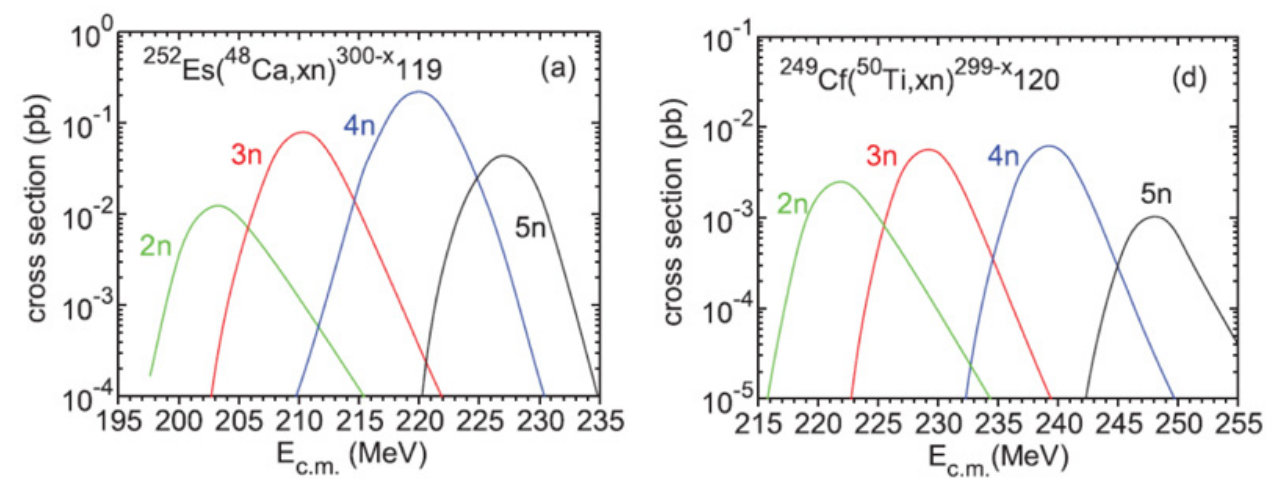

Figure 4. Synthesis cross sections of yet undiscovered superheavy nuclei of $Z=119$ and $Z=120$ predicted by using the fusion-by-diffusion model.

as obtained in the frame of the self-consistent theory [30], producing superheavy $Z=120$ nuclei should not pose any difficulties. We chose these two cases of fusion reactions for which our predictions are the most optimistic. For $Z=120$, it turned out to be the reaction $\left.{ }^{249} \mathrm{Cf}\left({ }^{50} \mathrm{Ti}, x n\right)\right)^{299-x} 120$. The corresponding excitation function is shown on the right hand side in Fig. 4. The largest cross section of about $6 \mathrm{fb}$ is expected for the $3 n$ and $4 n$ evaporation channels. One very important remark is required here: carrying out this reaction requires a change of the projectile from ${ }^{48} \mathrm{Ca}$ used successfully up to now to ${ }^{50} \mathrm{Ti}$ with which no hot superheavy-element synthesis was completed successfully. Generally, prospects for the synthesis of element $Z=120$ are considerably worse than those for $Z=119$. To obtain $Z=119$, our calculations prefer the ${ }^{252} \mathrm{Es}\left({ }^{48} \mathrm{Ca}, x n\right){ }^{300-x} 119$ reaction, for which a synthesis cross section of about $0.2 \mathrm{pb}$ in the $4 n$ channel at $E_{c . m} \simeq 220 \mathrm{MeV}$ is expected. This case is shown in the left panel in Fig. 4.

Closing this section we want to emphasize that the existing theoretical evaluations of the fission barriers differ significantly. Even the results of the two models based on the microscopic-macroscopic approach differ dramatically for some nuclei. Our calculations indicate, in contrast to the self-consistent mean-field studies, that fission barriers, which are still quite substantial for some $Z=118$ nuclei, become lower than $5.5 \mathrm{MeV}$ for $Z=126$. Quite recently we noted a dramatic divergence in calculated fission barriers [32]. A brief review of recent progress in theoretical studies on fission barriers and fission half-lives of even-even superheavy nuclei can be found in Ref. [31].

\section{4. $K$-isomerism and possible stability enhancement}

A recent theoretical study [33] of barriers within both the MM Woods-Saxon and Skyrme SLy6 Hartree-Fock plus BCS models do not predict much chance to produce even heavier $(Z>126)$ nuclei. Moreover, it seems that two current methods of making superheavy elements in the laboratory, cold and hot fusion reactions, seem to reach their limits. On the other hand, not all superheavy isotopes with $Z<118$ have been produced yet. Therefore, while pondering upon possible new reactions leading towards the island of stability, it may be worthwhile to search for long-lived exotic superheavy configurations. Obvious candidates are high- $K$ isomers, for which increased stability is expected due to some specific hindrance mechanisms. In our recent work [34] we went even further: from systematic calculations it turned out that some nuclei can have a ground state with a high- $K$ quantum number. 

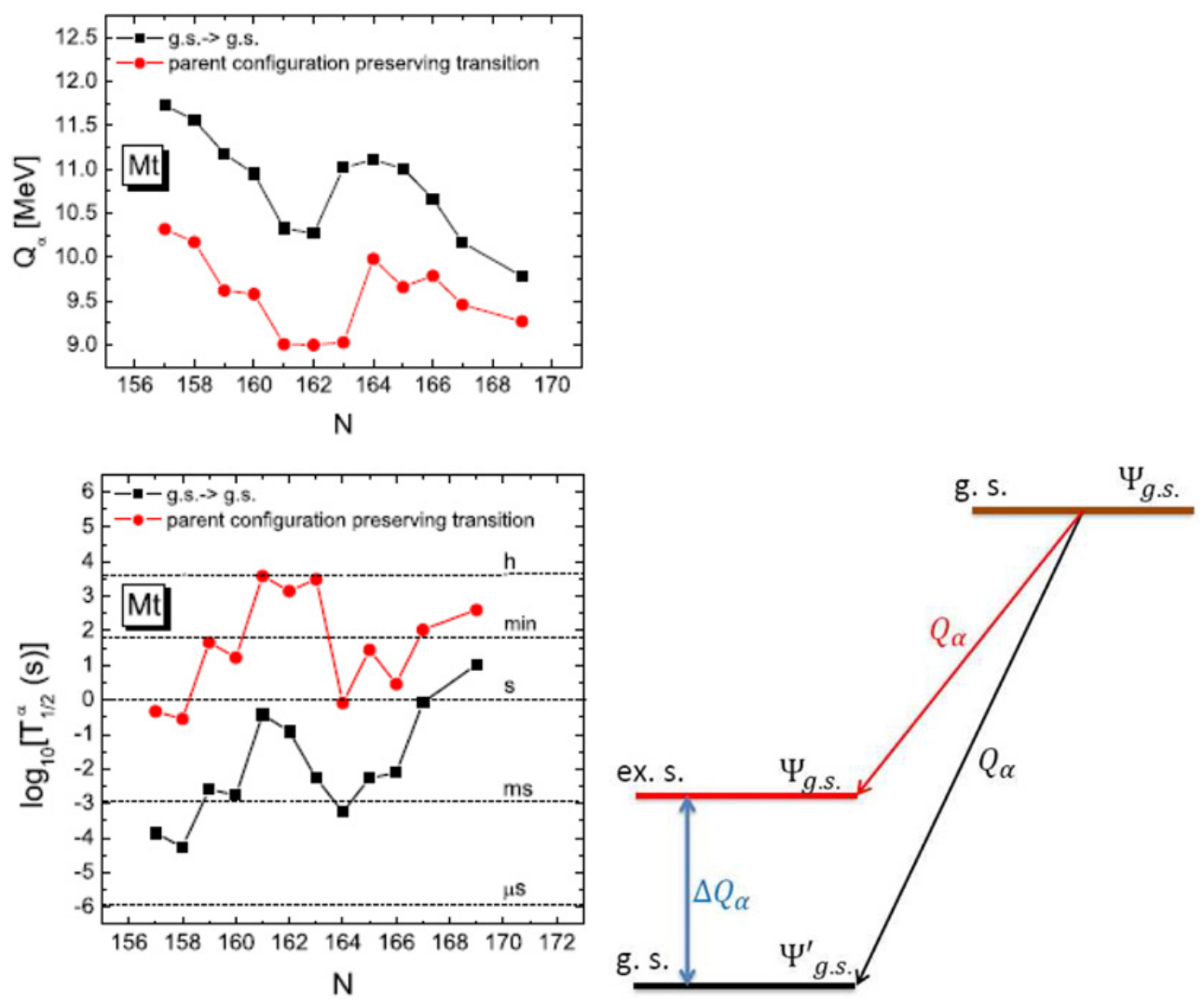

Figure 5. $Q_{\alpha}$ values calculated with blocking following from the WS model and the increase in $\alpha$ - half-life induced by $\Delta Q_{\alpha}$ [36], assuming a complete decay hindrance to different configurations (left panel). Schematic representation of appropriate transitions (right panel).

The structure of odd-odd nuclei is typically more complicated than that of odd- $A$ systems. If we disregard collective vibrations, the ground-state configuration is a result of coupling the unpaired neutron and proton to a total angular momentum. The energy ordering of coupled configurations is usually attributed to a residual neutron-proton interaction. This is why the investigation of odd-odd nuclei requires a significant expansion of the used method. This was done in Ref. [35]. An $\alpha$-decay hindrance of a high- $K$ configuration is expected when the same configuration in the daughter has a sizable excitation. If configuration-changing transitions had been strictly forbidden, the hindrance would have been determined by this excitation $\Delta Q_{\alpha}$ which is schematically shown in Fig. 5 (right panel). Also in Fig. 5 (left panel), a decrease in energy release, $\Delta Q_{\alpha}$, and $\log _{10} T_{\alpha}$ calculated according to [36], for ground-state to groundstate and configuration-preserving transitions are shown for various Meitnerium isotopes. The latter half-life would correspond to an absolute hindrance of configuration-changing decays. For our favourite case, ${ }^{272} \mathrm{Mt}$, such hindrance reaches six orders of magnitude. This takes us from the lifetime of milliseconds to hours. Let us stress that the whole argument is based on both the presence of deformed semi-magic shells at $Z=108$ and $N=162$ and the position of high- $\Omega$ intruder orbitals just above that shell. 


\section{Summary}

It may be that the heaviest element that can be created and identified will simply be limited by our synthesis techniques. Predictions based on the FBD model are rather pessimistic, giving the cross sections at the level of femtobarn. Synthesis of elements $Z>118$ will require a projectile heavier than ${ }^{48} \mathrm{Ca}$ (as fermium, $Z=100$, cannot be used as a target, while einsteinium, $Z=99$, is extremely difficult), and such reactions have thus so far proven fruitless. Might it be possible, therefore, to obtain superheavy isotopes with a structure that slows down fission and $\alpha$ decay? Of particular interest would be half-lives of several seconds or even minutes, since this would make it possible to analyze the chemical properties of individual radioactive atoms. The $\alpha$-decay which dominates in the extreme superheavy region is delayed by differences in structure of parent and daughter configurations, resulting either from their very different deformations or a different angular momentum coupling of unpaired nucleons.

M.K. and J.S. were co-financed by the National Science Centre under Contract No. UMO2013/08/M/ST2/00257 (LEA COPIGAL). One of the authors (P.J.) was cofinanced by Ministry of Science and Higher Education: "Iuventus Plus" grant No. IP2014 016073.

\section{References}

[1] Yu.Ts. Oganessian and V.K. Utyonkov., Nucl. Phys. A944, 62 (2015).

[2] V.K. Utyonkov et al., Phys. Rev. C 92, 034609 (2015).

[3] G. Münzenberg, Nucl. Phys. A944, 5(2015).

[4] S. Hofmann et al., Eur. Phys. J. A48 62, (2012).

[5] J. Khuyagbaatar et al., Phys. Rev. Lett. 112, 172501 (2014).

[6] Yu.Ts. Oganessian et al., Phys. Rev. C 87, 057302 (2013).

[7] Yu.Ts. Oganessian et al., Phys. Rev. C 87, 054621, (2013).

[8] W.J. Światecki, K. Siwek-Wilczyńska, J. Wilczyński, Phys. Rev. C 71, 014602 (2005).

[9] H.A. Kramers, Physica 7, 284 (1940).

[10] H. Haken, Synergetics (Springer, Berlin, 1978).

[11] T. Cap, K. Siwek-Wilczyńska, J. Wilczyński, Phys. Rev. C 83, 054602 (2011).

[12] D. Boilley et al., Phys. Rev. C 84, 054608 (2011).

[13] Zu-Hua Liu and Jing-Dong Bao, Phys. Rev. C 81, 044606 (2010).

[14] T. Cap et al., Acta Phys. Pol. B43, 297 (2012).

[15] S. Ćwiok et al., Comp. Phys. Comm. 46, 379 (1987).

[16] V.M. Strutinski, Sov. J. Nucl. Phys.3, 449 (1966); Nucl. Phys. A95, 420 (1967).

[17] H.J. Krappe, J.R. Nix, A.J. Sierk, Phys. Rev. C 20, 992 (1979).

[18] I. Muntian, Z. Patyk, A. Sobiczewski, Acta Phys. Pol. B32, 691 (2001).

[19] P. Möller and A. Iwamoto, Phys. Rev. C 61, 047602 (2000).

[20] M. Kowal, P. Jachimowicz and A. Sobiczewski, Phys. Rev. C 82, 014303 (2010).

[21] M. Kowal, P. Jachimowicz, J. Skalski, arXiv:1203.5013, (2012).

[22] P. Jachimowicz, M. Kowal, J. Skalski, Phys. Rev. C 85, 034305 (2012).

[23] K. Siwek-Wilczyńska et al., Phys. Rev. C 86, 014611 (2012).

[24] P. Möller et al., Phys. Rev. C 79, 064304 (2009).

[25] P. Möller et al., At. Data Nucl. Data Tables 59, 185 (1995).

[26] S. Hofmann et al., Eur. Phys. J. A52, 180 (2016). 
[27] S. Hofmann, Russ. Chem. Rev. 78, 1123 (2009).

[28] Yu.Ts. Oganessian et al., Phys. Rev. C 79, 024603 (2009).

[29] K. Siwek-Wilczyńska, T. Cap, J. Wilczyński, Int. J. Mod. Phys. E19, 500 (2010).

[30] A. Staszczak, A. Baran, W. Nazarewicz, Phys. Rev. C 87 (2013) 024320.

[31] A. Baran et al., Nucl. Phys. A944, 442 (2015).

[32] W. Brodzinski et al., JPS Conf. Proc. 6, 020054 (2015).

[33] W. Brodzinski and J. Skalski, Phys. Rev. C 88, 044307 (2013).

[34] P. Jachimowicz, M. Kowal, J. Skalski, Phys. Rev. C 92, 044306 (2015).

[35] P. Jachimowicz, M. Kowal, J. Skalski, Phys. Rev. C 89, 024304 (2014).

[36] G. Royer, Nucl. Phys. A848, 279 (2010). 